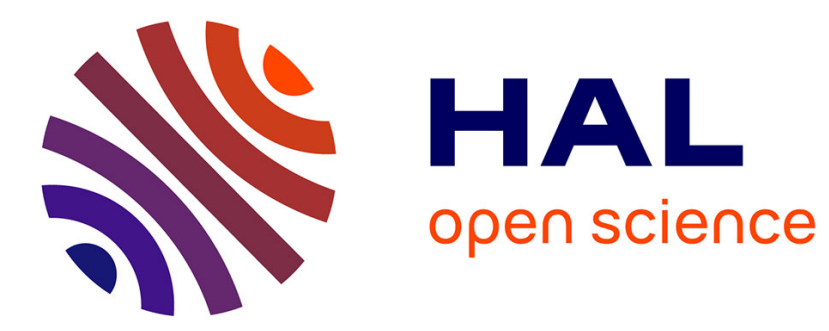

\title{
Extreme events in fiber based amplifiers
}

Kamal Hammani, Christophe Finot, Bertrand Kibler, John M. Dudley, Guy

Millot

\section{To cite this version:}

Kamal Hammani, Christophe Finot, Bertrand Kibler, John M. Dudley, Guy Millot. Extreme events in fiber based amplifiers. 11th International Conference on Transparent Optical Network, Jun 2009, Ponta Delgada, Portugal. pp.We.A1.4, 10.1109/ICTON.2009.5185178 . hal-00470024

\section{HAL Id: hal-00470024 https://hal.science/hal-00470024}

Submitted on 29 Jul 2010

HAL is a multi-disciplinary open access archive for the deposit and dissemination of scientific research documents, whether they are published or not. The documents may come from teaching and research institutions in France or abroad, or from public or private research centers.
L'archive ouverte pluridisciplinaire HAL, est destinée au dépôt et à la diffusion de documents scientifiques de niveau recherche, publiés ou non, émanant des établissements d'enseignement et de recherche français ou étrangers, des laboratoires publics ou privés. 


\title{
Extreme events in fiber based amplifiers
}

\author{
Kamal Hammani $^{1}$, Christophe Finot ${ }^{1}$, Bertrand Kibler ${ }^{1}$, John M. Dudley ${ }^{2}$ and Guy Millot ${ }^{1}$ \\ ${ }^{1}$ Institut Carnot de Bourgogne, UMR 5209 CNRS-Université de Bourgogne, 9 Av. A. Savary, Dijon, France \\ ${ }^{2}$ Institut FEMTO-ST, UMR 6174 CNRS-Université de Franche-Comté, Besançon, France \\ Tel: 33 (0)3 803959 26,e-mail: Christophe.Finot@u-bourgogne.fr
}

\begin{abstract}
We present experimental and theoretical results showing the emergence of rogue wave-like extreme intensity spikes during fiber-based amplification processes such as Raman effect or induced-modulational instability that rely on quasi-instantaneous gain. We outline that under certain circumstances, a partially incoherent pumping can induce large fluctuations of the amplified signal, and we propose various means to spectrally select the most extreme structures.
\end{abstract}

Keywords: extreme events, Raman amplification, parametric amplification.

\section{INTRODUCTION}

Optical fiber systems are well-known to provide convenient platforms with which to investigate a large and diverse set of fascinating fundamental nonlinear phenomena [1]. Optical telecommunications have therefore strongly benefited from all the potentiality offered by fibers, not only for ultra long haul high speed transmissions but also in the field of optical amplification. Erbium doped fiber amplifiers have indeed been a key ingredient of the telecommunication revolution of the last decade of the twentieth century. Two alternative amplification approaches based on optical fibers have also been developed based on the quasi-instantaneous gain provided by a strong pump through Raman amplification [2] or parametric amplification [3].

The practical applications of optical fibers have not been restricted to optical telecommunications, and a new generation of microstructured fibers enabling dispersion control combined with extreme confinement has been intensively used in the field of fibre supercontinuum generation. In this context, latest research developments have highlighted the possibility to observe in optical fibers very rare but intense optical events which have been termed rogue waves by analogy with hydrodynamics [4-7].

The question which we address here is concerned with the existence in telecommunication-based devices of such optical structures deviating strongly from a Gaussian probability distribution. We specifically investigate Raman and parametric amplifiers pumped by a partially incoherent field, and we will outline that under certain circumstances, rare but intense spikes can spontaneously emerge during the fiber amplification. We will carry out a temporal and spectral analysis of those extreme optical structures and we will propose strategies to isolate the rarest events.

\section{EXTREME EVENTS IN RAMAN AMPLIFIERS}

We first investigate a configuration based on a Raman fiber amplifier pumped by a partially incoherent wave.

\subsection{Experimental setup}

Our all-fiber experimental setup is sketched in Fig. 1 and relies exclusively on commercially available telecommunication-ready components. The pump is delivered by an unpolarized Raman fiber laser having a spectral width of a few tens of GHz. The temporal properties of this partially incoherent pump have been analyzed through autocorrelation measurements presented in Fig. 1(b). Experimental results are in good agreement with the calculated autocorrelation function (circles) based on the assumption of stochastic fluctuations with a Gaussian probability distribution [8]. The characteristic timescale of the fluctuations has been estimated at $25 \mathrm{ps}$, leading to rapid fluctuations of the pump signal as illustrated on the inset of Fig. 1(b).
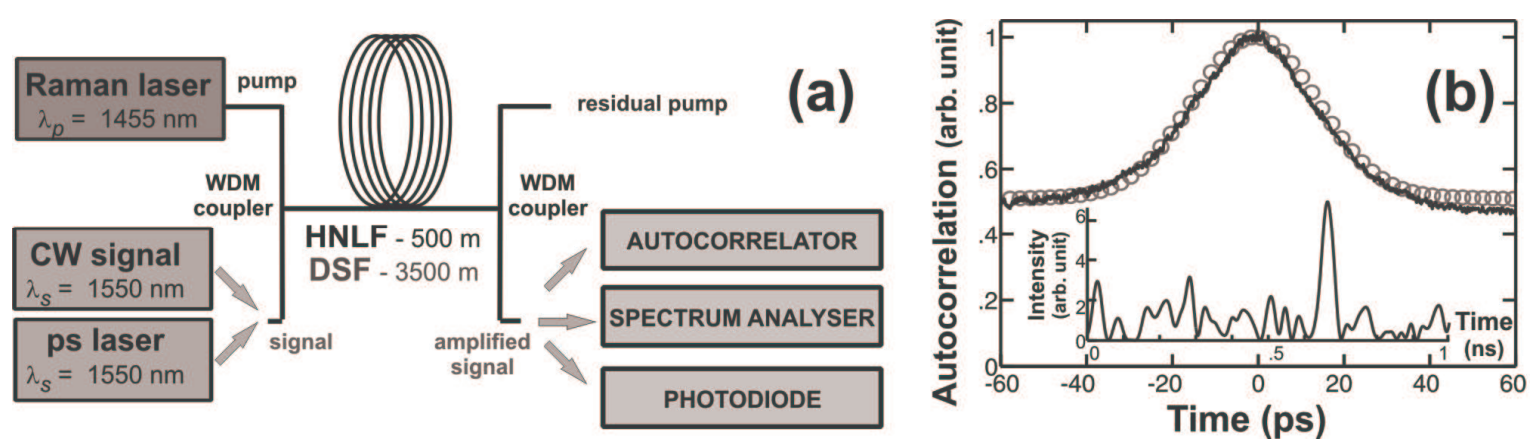

Figure 1. (a) Experimental setup. (b) Intensity autocorrelation signal of the pump signal. Inset : temporal fluctuations of the intensity profile of the pump. 
The pump and the signal to be amplified at $1550 \mathrm{~nm}$ are combined by a WDM coupler into a 500-m highly nonlinear fiber (HNLF) with nonlinear coefficient $\gamma=10 \mathrm{~W}^{-1} \cdot \mathrm{km}^{-1}$ and a second order dispersion $\beta_{2}=710^{-4} \mathrm{ps}^{2} . \mathrm{m}^{-1}$. We have also tested a 3500-m normal dispersion shifted fiber (DSF) with a higher dispersion of $3.210^{-3} \mathrm{ps}^{2} \cdot \mathrm{m}^{-1}$. Both fibers exhibit similar integrated Raman gain and cumulative nonlinearity. At the amplifier output, a second WDM coupler is used to isolate the signal which is then characterized by means of a photodiode, an optical spectrum analyzer and an optical intensity autocorrelator.

\subsection{Observations based on a continuous seed signal}

A typical measurement of an oscilloscope trace spanning 500ns using the HNLF-based amplifier is shown in Fig. 2(a) which illustrates that the amplified continuous signal exhibits some intense intensity spikes in the temporal domain. In order to better characterize the temporal properties of these features, we have also measured the intensity autocorrelation of the output signal for various values of gain (Fig. 2(b)). The results demonstrate for the HNLF a noticeable change in the contrast of the autocorrelation, highlighting significant differences in the pulse statistics. However, the temporal width of the structure is close to the characteristic time of the initial pump fluctuations. The output spectrum (Fig. 2(c)) has also been modified during the amplification process, with significant spectral broadening due to cross-phase modulation induced by the pump on the signal. The use of a fiber with a higher dispersion such as the DSF enables the suppression of such effects because of higher walk-off between the pump and the amplified signal.
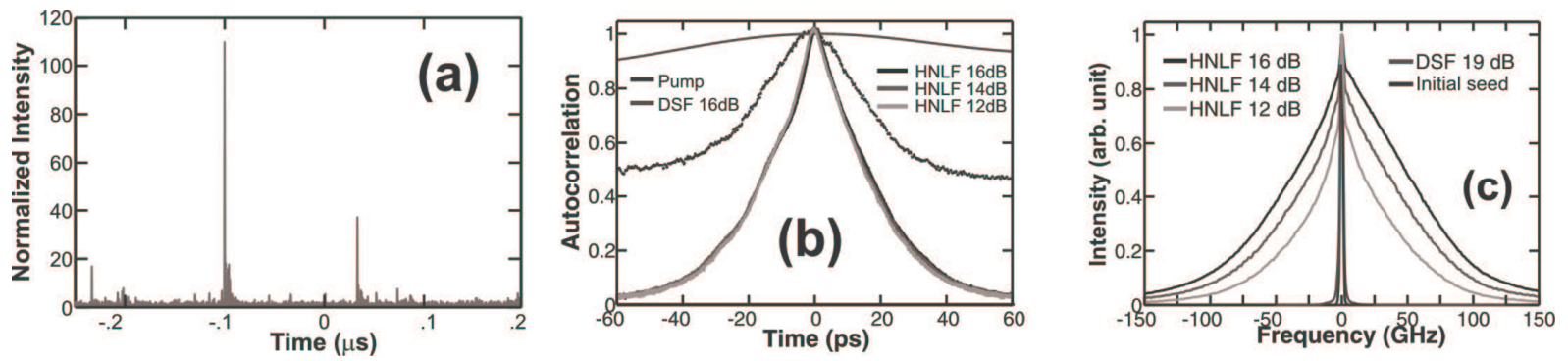

Figure 2. Temporal and spectral characterization of the amplified continuous signal amplified in the HNLF or DSF for various gains (a) Temporal signal recorded on a GHz photodiode for HNLF (b) Measured autocorrelation signal $\quad$ (c) Measured optical spectra.

\subsection{Observations based on a pulsed signal}

In order to gain some quantitative insights into the statistical distribution of the peak power fluctuations of the pulses, we have replaced the initial continuous signal by a picosecond pulse train delivered by a modelocked fiber laser. The eye diagrams recorded at the output of the Raman amplifier (Fig. 3(a)) show how very strong fluctuations have developed in the case of HNLF-based system. Measurements of the probability distribution of the peak powers after amplification further highlight the differences between the fibers. Indeed, for a HNLF, the probability distribution exhibits a long tail which is well-fitted by an exponentially decreasing function (when plotted on a semilogarithmic scale), which is typical of extreme value behavior. On the contrary, statistics for a DSF-amplifier can be described by a linear fit on this scale.

These experimental results have been confirmed by an intensive set of numerical simulations based on the nonlinear Schrödinger equation including Raman gain [1,2] : the linearly or exponentially decreasing tails have been quantitatively reproduced (Fig. 3(c)).
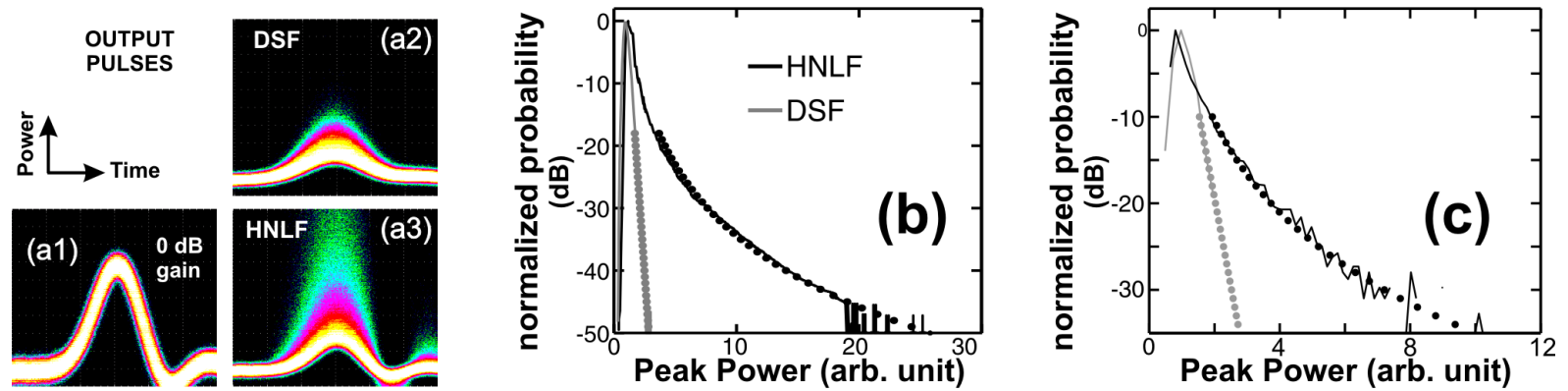

Figure 3.(a) Eye diagrams of the signal before and after a 3-dB amplification in a HNLF and a DSF (b) Statistical distribution of the peak powers obtained experimentally (c) Statistical distribution obtained from the numerical integration of the NLSE including the Raman gain. 


\subsection{Spectral selection of the rare events}

In order to better understand the dynamics of those extreme events, it is of much interest to develop techniques to usefully isolate such events. From a time-frequency representation of a pulse train (Fig. 4(a)), we can make out that the extreme events are associated with the largest spectral expansion. This suggests that an appropriate offset filtering method can be efficiently used in order to select the rarest events. Indeed, by using an optical bandpass filter (OBPF) centered on the signal central wavelength, a pulse train having the same repetition rate as the initial train is recovered as can been seen on Fig. 4(b1). On the contrary, for significant spectral offsets, only the most intense pulses are preserved (Fig. 4(b2)). Consequently, changing the spectral offset of the OBPF (Fig. $4(c))$ can provide an efficient way to control the number of the output pulses in the resulting random sequence.
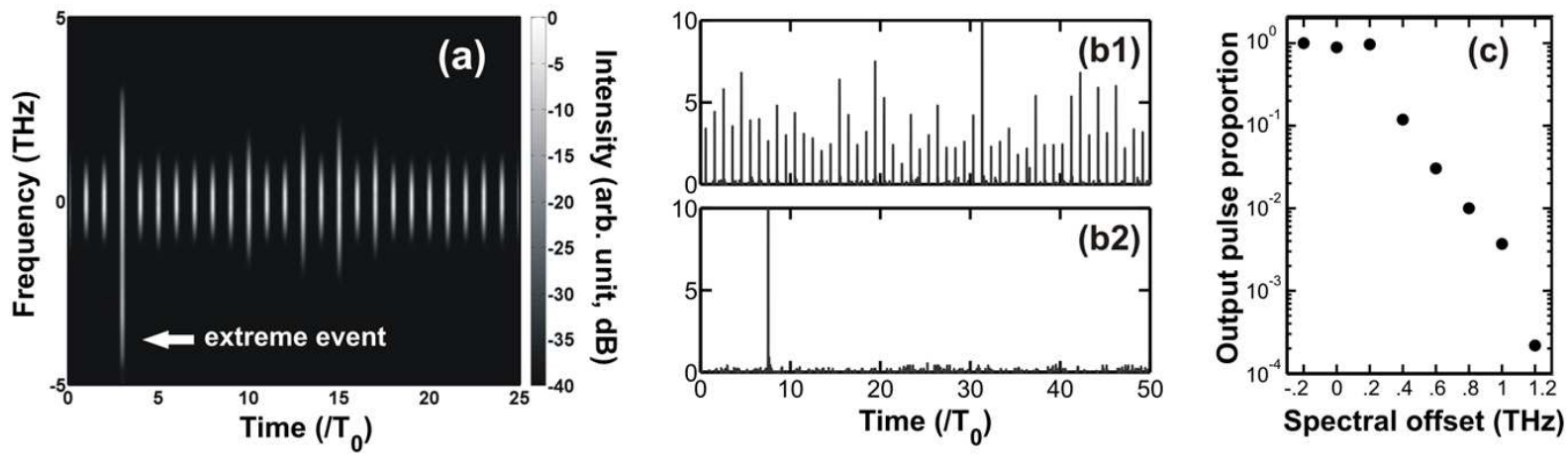

Figure 4. (a) Time-Frequency representation of the amplified pulse train $\quad$ (b) Temporal signal obtained after spectral slicing by a $100 \mathrm{GHz}$ filter offset by 0 and $1 \mathrm{THz}$ (subplots (b1) and (b2) respectively). $T_{0}$ is the initial period of the pulse train.

(c) Ratio between the number of initial pulses and the number of pulses detected after spectral filtering for various offset values.

\section{EXTREMES EVENTS IN PARAMETRIC AMPLIFIERS}

As Raman and parametric amplification are both based on a fast amplification processes which follows the rapid optical field fluctuations inherent to an incoherent wave, one may naturally wonder whether optical rogue events can also occur in modulation instability (MI)-based amplifier. In preliminary investigations, Sylvestre et al. have outlined that partially incoherent pumping, produced by fiber Raman lasers, could significantly deteriorate the quality of the amplified signals [9]. However, their analysis has been restricted to the frequency domain so that, to the best of our knowledge, the temporal profile and the associated statistical properties of an amplified signal have not been yet investigated in detail. We provide here experimental evidence of rare optical events in a parametric amplifier in presence of a strong partially incoherent pump.

\subsection{Experimental set-up}

Our experimental set-up used for MI-based amplification is close from the one described in Section 2.1. An initial incoherent pump is delivered by a home built source having a characteristic fluctuation time of 9 ps and a central wavelength of $1549 \mathrm{~nm}$ for an initial average power up to $110 \mathrm{~mW}$. The signal to be amplified is shifted by $\Omega$ compared to the pump wavelength. The amplification occurs in a 500-m highly nonlinear fiber having an anomalous dispersion of $-6 \quad 10^{-4} \mathrm{ps}^{2} \cdot \mathrm{m}^{-1}$ and a nonlinear coefficient of $10 \mathrm{~W}^{-1} \cdot \mathrm{km}^{-1}$. At the output of the amplifier, an OBPF enables us to remove the pump.

\subsection{Observation based on a continuous signal and spectral filtering}

Results of an initial continuous signal amplified in our device are plotted on Fig. 5(a). Similarly to the Raman case, intense spikes are visible on the oscilloscope trace. By changing $\Omega$, we have noticed that higher detuning leads to rarer and more intense structures. Our measurements have also confirmed that modulation instability based on a partially-incoherent pump lead to a higher gain coefficient as well as a broader amplification bandwidth compared to amplification relying on a coherent wave $[10,11]$ In addition,autocorrelation measurements has confirmed that initial ratio of 1:2 typical of the incoherent pump has significantly changed (Fig. 5(b)). Once again, all our experimental results are confirmed by numerical simulations based on the standard nonlinear Schrödinger equation. 

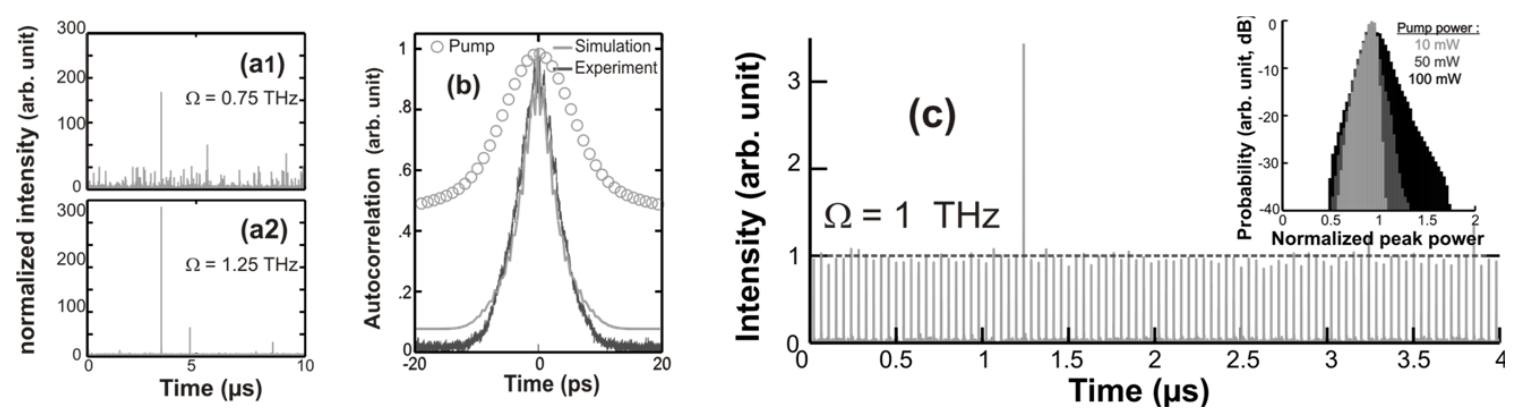

Figure 5. (a) Amplified signal recorded for different pump-signal offsets $\Omega$. (b) Experimental autocorrelation of the amplified signal (black line) compared to the numerical simulation results (grey line) and with the initial pump. (c) Pulse train after amplification. Inset : peak power statistical distribution for different pump powers.

\subsection{Observation based on a pulsed seed signal}

Similarly to Section 2.3 above, additional experiments have been carried out using a pulsed seed signal. When the pump-signal detuning $\Omega$ is fixed to $1 \mathrm{THz}$, most of the pulse train is not amplified as illustrated on Fig. 5(c). Only one shot is amplified and therefore deviates strongly from the average behavior. Related experimental statistical distributions recorded for different pump power levels are plotted on inset of Fig. 5(c). As the pump power increases, the probability distributions deviate from a purely Gaussian shape with the development of a tail towards higher peak powers.

\section{CONCLUSIONS}

Our results have shown that an amplification process based on a partially incoherent pump can lead to the emergence of intense signal peaks deviating significantly from the average dynamics. Both for parametric [11] and Raman [8] amplification, this temporal behavior can be explained by the instantaneous exponential transfer of the fluctuations of the pump to the signal. In both configurations, the rogue events can be isolated. These studies confirm that optical fiber devices at telecommunication wavelengths can be efficiently used as successful laboratory systems to understand rogue waves.

\section{ACKNOWLEDGEMENTS}

This work was supported by the Agence Nationale de la Recherche (ANR PERSYST II, SOFICARS and MANUREVA projects: ANR-07-TCOM-014, ANR-07-RIB-013-03 and ANR-08-SYSC-019), by the Conseil Régional de Bourgogne, and was carried out within the framework of the Research Networks GDR Phonomi2, COST action 299 FIDES and PRES UFC-UB.

\section{REFERENCES}

[1] G. P. Agrawal, Nonlinear Fiber Optics, Third Edition. San Francisco, CA: Academic Press, 2001.

[2] C. Headley and G. P. Agrawal, Raman amplification in fiber optical communications: Academic Press, 2005.

[3] R. H. Stolen and J. E. Bjorkholm, "Parametric amplification and frequency conversion in optical fibers," IEEE J. Quantum Electron., vol. 18, pp. 1062-1072, 1982.

[4] D. R. Solli, C. Ropers, P. Koonath, and B. Jalali, "Optical rogue waves," Nature, vol. 450, p. $1054,2007$.

[5] J. M. Dudley, G. Genty, and B. J. Eggleton, "Harnessing and control of optical rogue waves in supercontinuum generation " Opt. Express, vol. 16, pp. 3644-3651, 2008.

[6] B. Kibler, C. Finot, and J. M. Dudley, "Soliton and rogue wave statistics in supercontinuum generation in photonic crystal fibre with two zero dispersion wavelengths," European Journal of Physics, vol. in press, 2009.

[7] A. Mussot, A. Kudlinski, M. I. Kolobov, E. Louvergneaux, and M. Taki, "Convective instabilities and optical rogue waves in fibers with CW pumping," in LEOS Winter Topical Meeting, Innsbruck, 2009, p. TUC2.5.

[8] K. Hammani, C. Finot, J. M. Dudley, and G. Millot, "Optical rogue-wave fluctuations in fiber Raman amplifiers," Opt. Express, vol. 16, pp. 16467-16474, 2008.

[9] T. Sylvestre, A. Mussot, E. Lantz, and H. Maillote, "Impact of pump quality on the performances of fibre optical parametric amplifiers," in LEOS Winter Topical Meeting, Sorento, 2008, pp. 49-50.

[10] A. Sauter, S. Pitois, G. Millot, and A. Picozzi, "Incoherent modulation instability in instantaneous nonlinear Kerr media," Opt. Lett., vol. 30, pp. 2143-2145, 2005.

[11] K. Hammani, C. Finot, and G. Millot, "Emergence of extreme events in fiber-based parametric processes driven by a partially incoherent wave," Opt. Lett., vol. 34, pp. 1138-1140, 2009. 